\title{
Levitin-Polyak Well-Posedness in Vector Quasivariational Inequality Problems with Functional Constraints
}

\author{
J. Zhang, ${ }^{1}$ B. Jiang, ${ }^{2}$ and X. X. Huang ${ }^{3}$ \\ ${ }^{1}$ School of Mathematics and Physics, Chongqing University of Posts and Telecommunications, \\ Chongqing 400065, China \\ ${ }^{2}$ Department of Systems Engineering and Engineering Management, \\ The Chinese University of Hong Kong, Shatin, Hong Kong \\ ${ }^{3}$ School of Economics and Business Administration, Chongqing University, Chongqing 400030, China
}

Correspondence should be addressed to X. X. Huang, huangxuexiang@cqu.edu.cn

Received 17 March 2010; Accepted 6 July 2010

Academic Editor: Lai Jiu Lin

Copyright (C) 2010 J. Zhang et al. This is an open access article distributed under the Creative Commons Attribution License, which permits unrestricted use, distribution, and reproduction in any medium, provided the original work is properly cited.

We introduce several types of Levtin-Polyak well-posedness for a vector quasivariational inequality with functional constraints. Necessary and/or sufficient conditions are derived for them.

\section{Introduction}

It is well known that, under certain conditions, a Nash equilibrium problem can be formulated and solved as a variational inequality problem. A generalized Nash game is a Nash game in which each player's strategy depends on other players' strategies [1]. The connection between generalized Nash games and quasivariational inequalities was first recognized by Bensoussan [2]. Recently, some researchers $[1,3,4]$ found that mathematical models of many real world problems, including some engineering problems, can be formulated as certain kinds of variational inequality problems, including quasivariational inequality problems. However, as noted in [5], compared with variational inequality problems, the study on quasivariational inequality problems is still in its infancy, in particular only a few algorithms have been proposed to solve variational inequalities numerically.

Vector variational inequality problems were introduced by Giannessi [6] and are related to vector network equilibrium problems [7]. Since then, various types of vector 
variational inequalities were introduced and studied (see, e.g., $[8,9]$ and the references therein).

In this paper, we will consider vector quasivariational inequality problems with functional constraints, which are described below.

Let $(X,\|\cdot\|)$ be a normed space and $\left(Z, d_{1}\right)$ a metric space. Let $X_{1} \subseteq X, K \subseteq Z$ be nonempty and closed sets. Let $Y$ be a locally convex space and $C \subseteq Y$ be a nontrivial closed and convex cone with nonempty interior int $C$. Define the following order in $Y$, for any $y_{1}, y_{2} \in Y$,

$$
y_{1} \leq y_{2} \Longleftrightarrow y_{2}-y_{1} \in C \text {. }
$$

Let $L(X, Y)$ be the space of all the linear continuous operators from $X$ to $Y$. Let $F: X_{1} \rightarrow$ $L(X, Y)$ and $g: X_{1} \rightarrow Z$ be two functions. We denote by $\langle F(x), z\rangle$ the function value $F(x)(z)$, where $z \in X_{1}$. Let $S: X_{1} \rightarrow 2^{X_{1}}$ be a strict set-valued map (i.e., $S(x) \neq \emptyset$, for all $x \in X_{1}$ ).

Let

$$
X_{0}=\left\{x \in X_{1}: g(x) \in K\right\} .
$$

The vector quasivariational inequality problem with functional and abstract set constraints considered in this paper is:

$$
\begin{aligned}
& \text { Find } \bar{x} \in X_{0} \quad \text { such that } \bar{x} \in S(\bar{x}) \text { satisfying } \\
& \qquad\langle F(\bar{x}), x-\bar{x}\rangle \notin-\operatorname{int} C, \quad \forall x \in S(\bar{x}) .
\end{aligned}
$$

Denote by $\bar{X}$ the solution set of (VQVI).

Well-posedness for unconstrained and constrained optimization problems was first studied by Tikhonov [10] and Levitin and Polyak [11]. The issue being considered is that for each approximating solution sequence, there exists a subsequence that converges to a solution of the problem.

In Tikhonov's well posedness, the approximating solution is always feasible. However, it should be noted that many algorithms in optimization and variational inequalities, such as penalty-type methods and augmented Lagrangian methods, terminate when the constraint is approximately satisfied. These methods may generate sequences that may not be necessarily feasible [12].

Up to now, various extensions of these well posednesses have been developed and well studied (see, e.g., [13-18]). Studies on well posedness of optimization problems have been extended to vector optimization problems (see e.g., [19-24]). The study of LevitinPolyak well posedness for scalar convex optimization problems with functional constraints originates from [25]. Recently, this research was extended to nonconvex optimization problems with abstract and functional constraints [12] and nonconvex vector optimization problems with both abstract and functional constraints [26]. Well-posedness of variational inequality problems, mixed variational inequality problems, and equilibrium problems without functional constraints was investigated in the literature (see, e.g., [27-30]). Wellposedness in variational inequality problems with both abstract and functional constraints was investigated in [31]. Well-posedness of (generalized) quasivariational inequality and 
mixed quasivariational-like inequalities has been studied in the literature [32-35]. The study of well posedness for (generalized) vector variational inequality, vector quasiequilibria and vector equilibrium problems can be found in [36-39] and the references therein.

In this paper, we will introduce and study several types of Levitin-Polyak (LP in short) well posednesses and generalized LP well posednesses for vector quasivariational inequalities with functional constraints. The paper is organized as follows. In Section 2, four types of LP well posednesses and generalized LP well posednesses for vector quasivariational inequality problems will be defined. In Section 3, we will derive various criteria and characterizations for the various (generalized) LP well posednesses of constrained vector quasivariational inequalities.

\section{Definitions and Preliminaries}

Let $Z_{1}, Z_{2}$ be two normed spaces. A set-valued map $G$ from $Z_{1}$ to $2^{Z_{2}}$ is

(i) closed, on $Z_{3} \subseteq Z_{1}$, if for any sequence $\left\{x_{n}\right\} \subseteq Z_{3}$ with $x_{n} \rightarrow x \in Z_{3}$ and $y_{n} \in G\left(x_{n}\right)$ with $y_{n} \rightarrow y$, one has $y \in G(x)$;

(ii) lower semicontinuous (l.s.c. in short) at $x \in Z_{1}$, if $\left\{x_{n}\right\} \subseteq Z_{1}, x_{n} \rightarrow x$, and $y \in G(x)$ imply that there exists a sequence $\left\{y_{n}\right\} \subseteq Z_{2}$ satisfying $y_{n} \rightarrow y$ such that $y_{n} \in G\left(x_{n}\right)$ for $n$ sufficiently large. If $G$ is l.s.c. at each point of $Z_{1}$, we say that $G$ is l.s.c on $Z_{1}$.

Let $\left(P, d_{2}\right)$ be a metric space, $P_{1} \subseteq P$, and $p \in P$. In the sequel, we denote by $d_{P_{1}}(p)=$ $\inf \left\{d\left(p, p^{\prime}\right): p^{\prime} \in P_{1}\right\}$ the distance function from point $p$ to set $P_{1}$. For a topological vector space $V$, we denote by $V^{*}$ its dual space. For any cone $\Phi \subseteq V$, we will denote the (positive) polar cone of $\Phi$ by

$$
\Phi^{*}=\left\{\phi \in V^{*}: \phi(v) \geq 0, \forall v \in \Phi\right\}
$$

Let $e \in \operatorname{int} C$ be fixed. Denote

$$
C^{* 0}=\left\{\lambda \in C^{*}: \lambda(e)=1\right\} .
$$

Throughout this paper, we always assume that the feasible set $X_{0}$ is nonempty and the function $g$ is continuous on $X_{1}$.

Definition 2.1. (i) A sequence $\left\{x_{n}\right\} \subseteq X_{1}$ is called a type I Levtin-Polyak (LP in short) approximating solution sequence if there exists $\left\{\epsilon_{n}\right\} \subseteq R_{+}^{1}$ with $\epsilon_{n} \rightarrow 0$ such that

$$
\begin{gathered}
d_{X_{0}}\left(x_{n}\right) \leq \epsilon_{n}, \\
x_{n} \in S\left(x_{n}\right), \\
\left\langle F\left(x_{n}\right), x-x_{n}\right\rangle+\epsilon_{n} e \notin-\operatorname{int} C, \quad \forall x \in S\left(x_{n}\right) .
\end{gathered}
$$

(ii) $\left\{x_{n}\right\} \subseteq X_{1}$ is called a type II LP approximating solution sequence if there exist $\left\{\epsilon_{n}\right\} \subseteq R_{+}^{1}$ with $\epsilon_{n} \rightarrow 0$ and $\left\{y_{n}\right\} \subseteq X_{1}$ with $y_{n} \in S\left(x_{n}\right)$ such that (2.3)-(2.5) hold and

$$
\left\langle F\left(x_{n}\right), y_{n}-x_{n}\right\rangle-\epsilon_{n} e \in-C .
$$


(iii) $\left\{x_{n}\right\} \subseteq X_{1}$ is called a generalized type I LP approximating solution sequence if there exists $\left\{\epsilon_{n}\right\} \subseteq R_{+}^{1}$ with $\epsilon_{n} \rightarrow 0$ such that

$$
d_{K}\left(g\left(x_{n}\right)\right) \leq \epsilon_{n}
$$

and (2.4), (2.5) hold.

(iv) $\left\{x_{n}\right\} \subseteq X_{1}$ is called a generalized type II LP approximating solution sequence if there exist $\left\{\epsilon_{n}\right\} \subseteq R_{+}^{1}$ with $\epsilon_{n} \rightarrow 0$ and $\left\{y_{n}\right\} \subseteq X_{1}$ with $y_{n} \in S\left(x_{n}\right)$ such that (2.4)-(2.7) hold.

Definition 2.2. (VQVI) is said to be type I (resp., type II, generalized type I, generalized type II) LP well posed if the solution set $\bar{X}$ of (VQVI) is nonempty, and for any type I (resp., type II, generalized type I, generalized type II) LP approximating solution sequence $\left\{x_{n}\right\}$, there exist a subsequence $\left\{x_{n_{j}}\right\}$ of $\left\{x_{n}\right\}$ and $\bar{x} \in \bar{X}$ such that $x_{n_{j}} \rightarrow \bar{x}$.

Remark 2.3. (i) It is easily seen that if $Y=R^{1}, C=R_{+}^{1}$, then type I (resp., type II, generalized type I, generalized type II) LP well posedness of (VQVI) reduces to type I (resp., type II, generalized type I, generalized type II) LP well posedness of (QVI) defined in [34].

(ii) It is clear that any (generalized) type II LP approximating solution sequence is a (generalized) type I LP approximating solution sequence. Thus, (generalized) type I LP well posedness implies (generalized) type II LP well posedness. compact.

(iii)Each type of LP well posedness of (VQVI) implies that its solution set $\bar{X}$ is

To see that the various LP well posednesses of (VQVI) are adaptations of the corresponding LP well posednesses in minimizing problems by using the Auslender gap function, we consider the following general constrained optimization problem:

$$
\begin{array}{ll}
\min & f(x) \\
\text { s.t. } & x \in X_{1}^{\prime} \\
& g(x) \in K,
\end{array}
$$

where $X_{1}^{\prime} \subseteq X_{1}$ is nonempty and $f: X_{1} \rightarrow R^{1} \cup\{+\infty\}$ is proper. The feasible set of $(P)$ is $X_{0}^{\prime}$, where $X_{0}^{\prime}=\left\{x \in X_{1}^{\prime}: g(x) \in K\right\}$. The optimal set and optimal value of $(\mathrm{P})$ are denoted by $\bar{X}^{\prime}$ and $\bar{v}$, respectively. Note that if $\operatorname{Dom}(f) \cap X_{0}^{\prime} \neq \emptyset$, where

$$
\operatorname{Dom}(f)=\left\{x \in X_{1}: f(x)<+\infty\right\},
$$

then $\bar{v}<+\infty$. In this paper, we always assume that $\bar{v}>-\infty$. We note that LP well posedness for the special case, where $f$ is finite valued and l.s.c., $X_{1}^{\prime}$ is closed, has been studied in [12].

Definition 2.4. (i) A sequence $\left\{x_{n}\right\} \subseteq X_{1}^{\prime}$ is called a type I LP minimizing sequence for (P) if

$$
\begin{gathered}
\limsup _{n \rightarrow+\infty} f\left(x_{n}\right) \leq \bar{v}, \\
d_{X_{0}^{\prime}}\left(x_{n}\right) \longrightarrow 0 .
\end{gathered}
$$


Fixed Point Theory and Applications

(ii) $\left\{x_{n}\right\} \subseteq X_{1}^{\prime}$ is called a type II LP minimizing sequence for $(\mathrm{P})$ if

$$
\lim _{n \rightarrow+\infty} f\left(x_{n}\right)=\bar{v}
$$

and (2.10) hold.

(iii) $\left\{x_{n}\right\} \subseteq X_{1}^{\prime}$ is called a generalized type I LP minimizing sequence for $(\mathrm{P})$ if

$$
d_{K}\left(g\left(x_{n}\right)\right) \longrightarrow 0
$$

and (2.9) hold.

(iv) $\left\{x_{n}\right\} \subseteq X_{1}^{\prime}$ is called a generalized type II LP minimizing sequence for (P) if (2.11) and (2.12) hold.

Definition 2.5. (P) is said to be type I (resp., type II, generalized type I, generalized type II) LP well posed if the solution set $\bar{X}^{\prime}$ of (P) is nonempty, and for any type I (resp., type II, generalized type I, generalized type II) LP minimizing sequence $\left\{x_{n}\right\}$, there exist a subsequence $\left\{x_{n_{j}}\right\}$ of $\left\{x_{n}\right\}$ and $\bar{x} \in \bar{X}^{\prime}$ such that $x_{n_{j}} \rightarrow \bar{x}$.

The Auslender gap function for (VQVI) is

$$
f(x)=\sup _{x^{\prime} \in S(x)^{\lambda \in C^{* 0}}} \frac{\lambda\left\langle F(x), x-x^{\prime}\right\rangle}{\lambda(e)}, \quad \forall x \in X_{1} .
$$

From Lemma 1.1 in [40], we know that $C^{* 0}$ is weak* compact. This fact combined with that $\lambda(e)=1$ when $\lambda \in C^{* 0}$ implies that

$$
f(x)=\sup _{x^{\prime} \in S(x)^{\lambda \in C^{* 0}}} \min _{\lambda}\left\langle F(x), x-x^{\prime}\right\rangle, \quad \forall x \in X_{1} .
$$

Recall the following nonlinear scalarization function (see, e.g., [9]):

$$
\xi: Y \longrightarrow R^{1}: \xi(y)=\min \left\{t \in R^{1}: y-t e \in-C\right\}
$$

It is known that $\xi$ is a continuous, (strictly) monotone (i.e., for any $y_{1}, y_{2} \in Y, y_{1}-y_{2} \in$ $C$ implies that $\xi\left(y_{1}\right) \geq \xi\left(y_{2}\right)$ and $y_{1}-y_{2} \in \operatorname{int} C$ implies that $\left.\xi\left(y_{1}\right)>\xi\left(y_{2}\right)\right)$, subadditive, and convex function. Moreover, for any $t \in R^{1}$, it holds that $\xi(t e)=t$. Furthermore, following the proof of [9, Proposition 1.44], we can prove that

$$
\xi(y)=\sup _{\lambda \in C^{* 0}} \frac{\lambda(y)}{\lambda(e)}=\max _{\lambda \in C^{* 0}} \lambda(y), \quad \forall y \in Y .
$$

Let $X_{2} \subseteq X$ be defined by

$$
X_{2}=\left\{x \in X_{1} \mid x \in S(x)\right\} .
$$


First we have the following lemma.

Lemma 2.6. Let $f$ be defined by (2.14), then

(i) $f(x) \geq 0$, for all $x \in X_{2} \cap X_{0}$,

(ii) $f(x)=0$ and $x \in X_{2} \cap X_{0}$ if and only if $x \in \bar{X}$.

Proof. (i) Let $x \in X_{2} \cap X_{0}$, then $x \in S(x)$. We let $x^{\prime}$ in (2.14) be equal to $x$, then $f(x) \geq 0$.

(ii) Assume that $f(x)=0$. Suppose to the contrary that $x \notin \bar{X}$, then, there exists $x_{0} \in$ $S(x)$ such that

$$
\left\langle F(x), x_{0}-x\right\rangle \in-\operatorname{int} C
$$

Thus,

$$
\lambda\left\langle F(x), x-x_{0}\right\rangle>0, \quad \forall \lambda \in C^{* 0} .
$$

It follows that

$$
\min _{\lambda \in C^{* 0}} \lambda\left\langle F(x), x-x_{0}\right\rangle>0
$$

Hence, $f(x)>0$, contradicting the assumption, so $x \in \bar{X}$. Conversely, assume that $x \in \bar{X}$, then we have

$$
x \in X_{2} \cap X_{0}, \quad\left\langle F(x), x^{\prime}-x\right\rangle \notin-\operatorname{int} C, \quad \forall x^{\prime} \in S(x) .
$$

As a result, for any $x^{\prime} \in S(x)$, there exists $\lambda \in C^{* 0}$ such that

$$
\lambda\left\langle F(x), x-x^{\prime}\right\rangle \leq 0
$$

It follows that $f(x) \leq 0$. This fact combined with (i) implies that $f(x)=0$.

In the rest of this paper, we set $X_{1}^{\prime}$ in $(\mathrm{P})$ equal to $X_{2}$. Note that if the set-valued map $S$ is closed on $X_{2}$, then $X_{1}^{\prime}$ is closed. By Lemma 2.6, $\bar{x} \in \bar{X}$ if and only if $\bar{x}$ minimizes $f(x)$ (defined by (2.26)) over $X_{0} \cap X_{2}$ with $f(\bar{x})=0$.

The following lemma establishes some relationship between LP approximating solution sequence and LP minimizing sequence.

Lemma 2.7. Let the function $f$ be defined by (2.14) as follows:

(i) $\left\{x_{n}\right\} \subseteq X_{1}$ is a sequence such that there exists $\left\{\epsilon_{n}\right\} \subseteq R_{+}^{1}$ with $\epsilon_{n} \rightarrow 0$ satisfying (2.4)(2.5) if and only if $\left\{x_{n}\right\} \subseteq X_{1}^{\prime}$ and (2.9) holds with $\bar{v}=0$.

(ii) $\left\{x_{n}\right\} \subseteq X_{1}$ is a sequence such that there exist $\left\{\epsilon_{n}\right\} \subseteq R_{+}^{1}$ with $\epsilon_{n} \rightarrow 0$ and $\left\{y_{n}\right\} \subseteq X_{1}$ with $y_{n} \in S\left(x_{n}\right)$ satisfying (2.4)-(2.6) if and only if $\left\{x_{n}\right\} \subseteq X_{1}^{\prime}$ and (2.11) holds with $\bar{v}=0$. 
Proof. (i) Let $\left\{x_{n}\right\} \subseteq X_{1}$ be any sequence, if there exists $\left\{\epsilon_{n}\right\} \subseteq R_{+}^{1}$ with $\epsilon_{n} \rightarrow 0$ satisfying (2.4)-(2.5), then we can easily verify that

$$
\left\{x_{n}\right\} \subseteq X_{1}^{\prime}, \quad f\left(x_{n}\right) \leq \epsilon_{n} .
$$

It follows that (2.9) holds with $\bar{v}=0$.

For the converse, let $\left\{x_{n}\right\} \subseteq X_{1}^{\prime}$ and (2.9) hold. We can see that $\left\{x_{n}\right\} \subseteq X_{1}$ and (2.4) hold. Furthermore, by (2.9), we have that there exists

$$
\left\{\epsilon_{n}\right\} \subseteq R_{+}^{1} \quad \text { with } \epsilon_{n} \longrightarrow 0
$$

such that

$$
f\left(x_{n}\right) \leq \epsilon_{n}
$$

That is,

$$
\sup _{x^{\prime} \in S\left(x_{n}\right)} \inf _{\lambda \in C^{* 0}} \lambda\left\langle F\left(x_{n}\right), x_{n}-x^{\prime}\right\rangle \leq \epsilon_{n}
$$

Now, we will show that (2.5) holds, otherwise there exists $x_{0} \in S\left(x_{n}\right)$ such that

$$
\left\langle F\left(x_{n}\right), x_{0}-x_{n}\right\rangle+\epsilon_{n} e \in-\operatorname{int} C
$$

As a result, for any $\lambda \in C^{* 0}, \lambda\left\langle F\left(x_{n}\right), x_{n}-x_{0}\right\rangle>\epsilon_{n}$. Since $C^{* 0}$ is a weak ${ }^{*}$ compact set, we have

$$
\inf _{\lambda \in C^{* 0}} \lambda\left\langle F\left(x_{n}\right), x_{n}-x_{0}^{\prime}\right\rangle>\epsilon_{n}
$$

which contradicts (2.26).

(ii) Let $\left\{x_{n}\right\} \subseteq X_{1}$ be any sequence, we can check that

$$
\liminf _{n \rightarrow+\infty} f\left(x_{n}\right) \geq 0
$$

holds if and only if there exists $\left\{\alpha_{n}\right\} \subseteq R_{+}^{1}$ with $\alpha_{n} \rightarrow 0$ and $\left\{y_{n}\right\} \subseteq X_{1}$ with $y_{n} \in S\left(x_{n}\right)$ such that (2.6) (with $\epsilon_{n}$ replaced by $\alpha_{n}$ ) holds. From the proof of (i), we know that

$$
\limsup _{n \rightarrow+\infty} f\left(x_{n}\right) \leq 0
$$

and $\left\{x_{n}\right\} \subseteq X_{1}^{\prime}$ hold if and only if $\left\{x_{n}\right\} \subseteq X_{1}$ such that there exists $\left\{\beta_{n}\right\} \subseteq R_{+}^{1}$ with $\beta_{n} \rightarrow$ 0 satisfying (2.4)-(2.5) (with $\epsilon_{n}$ replaced by $\beta_{n}$ ). Finally, we set $\epsilon_{n}=\max \left\{\alpha_{n}, \beta_{n}\right\}$ and the conclusion follows.

The next proposition establishes relationships between the various LP well posednesses of (VQVI) and those of $(\mathrm{P})$ with $f(x)$ defined by (2.14). 
Proposition 2.8. Assume that $\bar{X} \neq \emptyset$, then

(i) (VQVI) is generalized type I (resp., generalized type II) LP well posed if and only if (P) is generalized type I (resp., generalized type II) LP well posed with $f(x)$ defined by (2.14).

(ii) If (VQVI) is type I (resp., type II) LP well posed, (P) is type I (resp., type II) LP well posed with $f(x)$ defined by (2.14).

Proof. By Lemma 2.6, if $\bar{X} \notin \emptyset, \bar{x}$ is a solution of (VQVI) if and only if $\bar{x}$ is an optimal solution of $(\mathrm{P})$ with $\bar{v}=f(\bar{x})=0$ and $f(x)$ defined by $(2.14)$.

(i) Similar to the proof of Lemma 2.7, it is also routine to check that a sequence $\left\{x_{n}\right\}$ is a generalized type I (resp., generalized type II) LP approximating solution sequence if and only if it is a generalized type I (resp., generalized type II) LP minimizing sequence of (P). So (VQVI) is generalized type I (resp., generalized type II) LP well posed if and only if (P) is generalized type I (resp., generalized type II) LP well posed with $f(x)$ defined by (2.26).

(ii) Since $X_{0}^{\prime} \subseteq X_{0}, d_{X_{0}}(x) \leq d_{X_{0}^{\prime}}(x)$ for any $x$. This fact together with Lemma 2.7 implies that a type I (resp., type II) LP minimizing sequence of (P) is a type I (resp., type II) LP approximating solution sequence. So type I (resp., type II) LP well posedness of (VQVI) implies type I (resp., type II) LP well posedness of (P) with $f(x)$ defined by (2.26).

To end this section, we note that all the results in [12] for the well posedness hold for (P) so long as $X_{1}^{\prime}$ is closed, $f$ is l.s.c. on $X_{1}^{\prime}$, and $\operatorname{Dom}(f) \cap X_{0}^{\prime} \neq \emptyset$.

\section{Criteria and Characterizations for Various LP Well-Posedness of (VQVI)}

In this section, we give necessary and/or sufficient conditions for the various types of (generalized) LP well posednesses defined in Section 2.

Consider the following statement:

$[\bar{X} \neq \emptyset$ and for any type I (resp., type II, generalized type I, generalized type II) LP

approximating solution sequence $\left\{x_{n}\right\}$, we have $d_{\bar{X}}\left(x_{n}\right) \longrightarrow 0$ ].

The next proposition can be straightforwardly proved.

Proposition 3.1. If (VQVI) is type I (resp., type II, generalized type I, generalized type II) LP well posed, then (3.1) holds. Conversely, if (3.1) holds and $\overline{\mathrm{X}}$ is compact, then (VQVI) is type I (resp., type II, generalized type I, generalized type II) LP well posed.

Now, we consider a real-valued function $c=c(t, s, r)$ defined for $t, s, r \geq 0$ sufficiently small such that

$$
\begin{gathered}
c(t, s, r) \geq 0, \quad \forall t, s, r, c(0,0,0)=0, \\
s_{n} \longrightarrow 0, t_{n} \geq 0, r_{n}=0, \quad c\left(t_{n}, s_{n}, r_{n}\right) \longrightarrow 0 \quad \text { imply that } t_{n} \longrightarrow 0 .
\end{gathered}
$$


With the help of Lemma 2.7, analogously to [35, Theorems 3.1, and 3.2], we can prove the following two theorems.

Theorem 3.2. If (VQVI) is type II LP well posed, the set-valued map $S$ is closed valued, then there exists a function c satisfying (3.2) such that

$$
|f(x)| \geq c\left(d_{\bar{X}}(x), d_{X_{0}}(x), d_{S(x)}(x)\right) \quad \forall x \in X_{1}
$$

where $f(x)$ is defined by (2.14). Conversely, suppose that $\bar{X}$ is nonempty and compact, and (3.3) holds for some $c$ satisfying (3.2), then (VQVI) is type II LP well posed.

Theorem 3.3. If (VQVI) is type II LP well posed in the generalized sense, the set-valued mapping $S$ is closed, then there exists a function c satisfying (3.2) such that

$$
|f(x)| \geq c\left(d_{\bar{X}}(x), d_{K}(g(x)), d_{S(x)}(x)\right) \quad \forall x \in X_{1}
$$

where $f(x)$ is defined by (2.14). Conversely, suppose that $\bar{X}$ is nonempty and compact, and (3.4) holds for some $c$ satisfying (3.2), then (VQVI) is generalized type II LP well posed.

Next we give Furi-Vignoli type characterizations [41] for the (generalized) type I LP well posednesses of (VQVI).

Let $(X,\|\cdot\|)$ be a Banach space. Recall that the Kuratowski measure of noncompactness for a subset $H$ of $X$ is defined as

$$
\mu(H)=\inf \left\{\epsilon>0: H \subseteq \bigcup_{i=1}^{n} H_{i}, \operatorname{diam}\left(H_{i}\right)<\epsilon, i=1, \ldots, n\right\}
$$

where diam $\left(H_{i}\right)$ is the diameter of $H_{i}$ defined by

$$
\operatorname{diam}\left(H_{i}\right)=\sup \left\{\left\|x_{1}-x_{2}\right\|: x_{1}, x_{2} \in H_{i}\right\}
$$

For any $\epsilon \geq 0$, define

$$
\begin{gathered}
\Psi_{1}(\epsilon)=\left\{x \in X_{1}^{\prime}: f(x) \leq \bar{v}+\epsilon, d_{X_{0}^{\prime}}(x) \leq \epsilon\right\}, \\
\Psi_{2}(\epsilon)=\left\{x \in X_{1}^{\prime}: f(x) \leq \bar{v}+\epsilon, d_{K}(g(x)) \leq \epsilon\right\} .
\end{gathered}
$$

Lemma 3.4. Let $f(x)$ be defined by (2.14) and $\bar{v}=0$. Let

$$
\begin{gathered}
\Omega_{1}(\epsilon)=\left\{x \in X_{1}: x \in S(x), d_{X_{0}}(x) \leq \epsilon,\left\langle F(x), x^{\prime}-x\right\rangle+\epsilon e \notin-\operatorname{int} C, \forall x^{\prime} \in S(x)\right\}, \\
\Omega_{2}(\epsilon)=\left\{x \in X_{1}: x \in S(x), d_{K}(g(x)) \leq \epsilon,\left\langle F(x), x^{\prime}-x\right\rangle+\epsilon e \notin-\operatorname{int} C, \forall x^{\prime} \in S(x)\right\},
\end{gathered}
$$

then one has $\Psi_{1}(\epsilon) \subset \Omega_{1}(\epsilon)$ and $\Psi_{2}(\epsilon)=\Omega_{2}(\epsilon)$. 
Proof. First, we prove the former result. For any $x \in X_{1}^{\prime}$ satisfying

$$
f(x) \leq \epsilon, \quad d_{X_{0}^{\prime}}(x) \leq \epsilon,
$$

we have $x \in X_{1}$ and $x \in S(x)$. We will show that $\left\langle F(x), x^{\prime}-x\right\rangle+\epsilon e \notin-\operatorname{int} C$, for all $x^{\prime} \in$ $S(x)$. Otherwise, there exists $x^{\prime} \in S(x)$ such that $\left\langle F(x), x^{\prime}-x\right\rangle+\epsilon e \in-\operatorname{int} C$. By the weak ${ }^{*}$ compactness of $C^{* 0}$, we have $\inf _{\mathcal{L} \in C^{* 0}} \lambda\left\langle F(x), x-x^{\prime}\right\rangle>\epsilon$, which leads to $f(x)>\epsilon$ and gives rise to a contradiction. Furthermore, we observe that $X_{0}^{\prime} \subseteq X_{0}$. This fact combined with $d_{X_{0}^{\prime}}(x) \leq \epsilon$ implies that $d_{X_{0}}(x) \leq \epsilon$.

Now, we prove the equivalence between $\Psi_{2}(\epsilon)$ and $\Omega_{2}(\epsilon)$. Firstly, we can establish the same inclusion for $\Psi_{2}(\epsilon)$ and $\Omega_{2}(\epsilon)$ analogously to the proof stated above. Then if $x \in X_{1}$ satisfies $x \in S(x), d_{K}(x) \leq \epsilon$ and

$$
\left\langle F(x), x^{\prime}-x\right\rangle+\epsilon e \notin-\operatorname{int} C, \quad \forall x^{\prime} \in S(x) .
$$

It is routine to check that $x \in X_{1}^{\prime}$. From (3.11), we know that for each $x^{\prime} \in S(x)$, there exists $\lambda \in C^{* 0}$ such that $\lambda\left\langle F(x), x-x^{\prime}\right\rangle \leq \epsilon$. As a result, we can see that $f(x) \leq \epsilon$. Thus, we prove the conclusion.

The next lemma can be proved analogously to ([25, Theorem 5.5]).

Lemma 3.5. Let $(X,\|\cdot\|)$ be a Banach space. Suppose that $f$ is l.s.c. on $X_{1}^{\prime}$ and bounded below on $X_{0}^{\prime}$. Assume that the optimal solution set of $(\mathrm{P})$ is nonempty and compact, then, $(\mathrm{P})$ is (generalized) type I LP well posed if and only if

$$
\left(\lim _{\epsilon \rightarrow 0} \mu\left(\Psi_{2}(\epsilon)\right)=0 .\right) \lim _{\epsilon \rightarrow 0} \mu\left(\Psi_{1}(\epsilon)\right)=0 .
$$

To continue our study, we make some assumptions below.

Assumption 1. (i) $X$ is a Banach space.

(ii) The set-valued map $S$ is closed, and lower semicontinuous on $X_{1}$.

(iii) The map $F$ is continuous on $X_{1}$.

We have the following lemma concerning the 1.s.c. of $f$ defined by (2.14).

Lemma 3.6. Let function $f$ be defined by (2.14) and Assumption 1 hold, then $f$ is l.s.c. function from $X_{1}$ to $R^{1} \cup\{+\infty\}$. Further assume that the solution set $\bar{X}$ of $(\mathrm{VQVI})$ is nonempty, then $\operatorname{Dom}(f) \neq \emptyset$.

Proof. First we show that $f(x)>-\infty$, for all $x \in X_{1}$. Suppose to the contrary that there exists $x_{0} \in X_{1}$ such that $f\left(x_{0}\right)=-\infty$, then,

$$
\inf _{\lambda \in C^{* 0}} \lambda\left(\left\langle F\left(x_{0}\right), x_{0}-x\right\rangle\right)=-\infty, \quad \forall x \in S\left(x_{0}\right)
$$

That is,

$$
\sup _{\lambda \in C^{* 0}} \lambda\left(\left\langle F\left(x_{0}\right), x_{0}-x\right\rangle\right)=+\infty, \quad \forall x \in S\left(x_{0}\right)
$$


Namely,

$$
\xi\left(\left\langle F\left(x_{0}\right), x_{0}-x\right\rangle\right)=+\infty, \quad \forall x \in S\left(x_{0}\right),
$$

which is impossible since $\xi$ is a finite function on $Y$. Second, we show that $f$ is l.s.c. on $X_{1}$. Note that the function

$$
h(x, y)=\min _{\lambda \in C^{* 0}} \lambda(\langle F(x), x-y\rangle)=-\xi(\langle F(x), y-x\rangle)
$$

is continuous on $X_{1} \times X_{2}$ by the continuity of $F$ on $X_{1}$ and the continuity of $\xi$. We also note that $f(x)=\sup _{y \in S(x)} h(x, y)$. Let $t \in R^{1}$. Suppose that the sequence $\left\{x_{n}\right\} \subseteq X_{1}$ satisfies

$$
f\left(x_{n}\right) \leq t
$$

and $x_{n} \rightarrow x^{*} \in X_{1}$. For any $y \in S\left(x^{*}\right)$, by the lower semicontinuity of $S$ and continuity of $h$, we have a sequence $\left\{y_{n}\right\}$ with $y_{n} \in S\left(x_{n}\right)$ converging to $y$ such that

$$
h\left(x^{*}, y\right)=\lim _{n \rightarrow+\infty} h\left(x_{n}, y_{n}\right) \leq \liminf _{n \rightarrow+\infty} f\left(x_{n}\right) \leq t
$$

It follows that $f\left(x^{*}\right)=\sup _{y \in S(x)} h\left(x^{*}, y\right) \leq t$. Hence, $f$ is 1.s.c. on $X_{1}$. Furthermore, if $\bar{X} \neq \emptyset$, by Lemma 2.6, we see that $\operatorname{Dom}(f) \neq \emptyset$.

Theorem 3.7. Let Assumption 1 hold and let the solution set $\bar{X}$ of (QVVI) be nonempty and compact, then, (VQVI) is generalized type I LP well posed if and only if

$$
\lim _{\epsilon \rightarrow 0} \mu\left(\Omega_{2}(\epsilon)\right)=0
$$

Proof. Note that the function $f(x)$ defined by (2.14) is nonnegative on $X_{0}^{\prime}$. By the lower semicontinuity of $S$ and Lemma 3.6, $f$ is l.s.c. on $X_{1}^{\prime}=X_{1} \cap X_{2}$. Moreover, $X_{1}^{\prime}$ is closed, since $S$ is closed on $X_{1} \cap X_{2}$. By Proposition 2.8, Lemmas 3.4 and 3.5, the conclusion follows.

Although the type I (type II) LP well posedness of (VQVI) is not equivalent to the type I (type II) LP well posedness of (P), we can still establish the same characterization for type I (type II) LP well posedness of (VQVI) as Theorem 3.7. We need the next lemma.

Lemma 3.8. Let Assumption 1 hold, then $\Omega_{1}(\epsilon)$ defined by (3.8) is closed.

Proof. Let $x_{n} \in \Omega_{1}(\epsilon)$ and $x_{n} \rightarrow x_{0}$. We show that $x_{0} \in \Omega_{1}(\epsilon)$. It is obvious that $d_{\mathrm{X}_{0}}\left(x_{0}\right) \leq \epsilon$. Since $x_{n} \in S\left(x_{n}\right)$ and $x_{n} \rightarrow x_{0}$, by the closedness of $S$, we have $x_{0} \in S\left(x_{0}\right)$. Moreover, since

$$
\left\langle z_{n}, x^{\prime}-x_{n}\right\rangle+\epsilon e \notin-\operatorname{int} C, \quad \forall x^{\prime} \in S\left(x_{n}\right)
$$


hold and $S$ is 1.s.c., for any $y \in S\left(x_{0}\right)$, we can find that $y_{n} \in S\left(x_{n}\right)$ with $\left\{y_{n}\right\} \rightarrow y$ such that

$$
\left\langle F\left(x_{0}\right), y-x_{0}\right\rangle=\lim _{n \rightarrow \infty}\left\langle F\left(x_{n}\right), y_{n}-x_{n}\right\rangle+\epsilon e \notin-\operatorname{int} C .
$$

Hence, $\Omega_{1}$ is closed.

Theorem 3.9. Let Assumption 1 hold and let $\Omega_{1}(\epsilon)$ be defined by (2.14). Assume that the solution set $\bar{X}$ of (QVVI) is nonempty and compact, then (VQVI) is type I LP well posed if and only if

$$
\lim _{\epsilon \rightarrow 0} \mu\left(\Omega_{1}(\epsilon)\right)=0
$$

Proof. The proof is similar to that of Theorem 3.4 in [35] and thus omitted.

Example 3.10. (i) Let $X=Y=R^{2}, C=R_{+}^{2} e=(1,1)^{T}, X_{1}=R^{2}$, and $X_{0}=R_{+}^{2} . F$ maps $R_{+}^{2}$ into an identical mapping, that is to say $\langle F(x), y\rangle=\left(y_{1}, y_{2}\right)^{T}$, for any $x, y \in R^{2}$. The set valued mapping $S$ is defined as follows, given $y \in S(x)$ for some $x, y \in R^{2}$, then

$$
y_{i}= \begin{cases}{\left[x_{i}, 1\right],} & \text { if } x_{i} \leq 1 \\ {\left[2 x_{i}-1,3 x_{i}-2\right],} & \text { if } x_{i}>1\end{cases}
$$

with $i \in\{1,2\}$, of course $S$ is closed and 1.s.c. Now, we can show that, when $0 \leq \epsilon \leq 1$, $\Omega_{1}(\epsilon) \subseteq\left\{x \mid-\epsilon \leq x_{1} \leq 1,-\epsilon \leq x_{2} \leq 1\right\}$, which is bounded. Thus, $\mu(\Omega(\epsilon))=0$, by applying Theorem 3.9, we know that (VQVI) is type I LP well posed.

(i) Suppose that $G$ is a set-valued mapping from $R^{2}$ to $2^{R^{2}}$, for fixed $x \in R^{2}, y \in G(x)$ implies that

$$
y_{i}= \begin{cases}{\left[x_{i}, 1\right],} & \text { if } x_{i} \leq 1 \\ {\left[1,2 x_{i}-1\right],} & \text { if } x_{i}>1\end{cases}
$$

with $i \in\{1,2\}$, obviously $G$ is still closed and l.s.c. If we replace $S$ by $G$ in (i), then $\Omega_{1}(\epsilon) \supseteq\{x \mid$ $\left.-\epsilon \leq x_{1} \leq 1+\epsilon, x_{2} \geq 0\right\}$ with $0 \leq \epsilon \leq 1$, which is unbounded. Therefore, $\lim _{\epsilon \rightarrow 0} \mu\left(\Omega_{1}(\epsilon)\right) \neq 0$ and the (VQVI) is not LP well posed in sense of type I. Actually, the solution set of this problem is $\left\{x \mid 0 \leq x_{1} \leq 1, x_{2} \geq 0\right\} \cup\left\{x \mid 0 \leq x_{2} \leq 1, x_{1} \geq 0\right\}$ and thus unbounded.

Definition 3.11. (i) Let $Z$ be a topological space, and let $Z_{1} \subseteq Z$ be nonempty. Suppose that $h: Z \rightarrow R^{1} \cup\{+\infty\}$ is an extended real-valued function. $h$ is said to be level compact on $Z_{1}$ if, for any $s \in R^{1}$, the subset $\left\{z \in Z_{1}: h(z) \leq s\right\}$ is compact.

(ii) Let $Z$ be a finite dimensional normed space, and let $Z_{1} \subseteq Z$ be nonempty. A function $h: Z \rightarrow R^{1} \cup\{+\infty\}$ is said to be level bounded on $Z_{1}$ if $Z_{1}$ is bounded or

$$
\lim _{z \in Z_{1},\|z\| \rightarrow+\infty} h(z)=+\infty
$$


The following proposition presents some sufficient conditions for type I LP well posedness of (VQVI)

Proposition 3.12. Let Assumption 1 hold. Further assume that one of the following conditions holds.

(i) There exists $0<\delta_{1}<\delta_{0}$ such that $X_{1}\left(\delta_{1}\right)$ is compact, where

$$
X_{1}\left(\delta_{1}\right)=\left\{x \in X_{1} \cap X_{2}: d_{X_{0}}(x) \leq \delta_{1}\right\}
$$

(ii) the function $f$ defined by (2.14) is level compact on $X_{1} \cap X_{2}$,

(iii) $\mathrm{X}$ is finite dimensional and

$$
\lim _{x \in X_{1} \cap X_{2},\|x\| \rightarrow+\infty} \max \left\{f(x), d_{X_{0}}(x)\right\}=+\infty,
$$

where $f$ is defined by (2.14).

(iv) There exists $0<\delta_{1}<\delta_{0}$ such that $f$ is level-compact on $X_{1}\left(\delta_{1}\right)$ defined by (3.26). Then, (VQVI) is type I LP well posed.

Proof. First, we show that each one of (i), (ii), and (iii) implies (iv). Clearly, either of (i) and (ii) implies (iv). Now, we show that (iii) implies (iv). We notice that the set $X_{1} \cap X_{2}$ is closed by the closedness of $S$. Then, we need only to show that for any $t \in R^{1}$, the set

$$
A=\left\{x \in X_{1}\left(\delta_{1}\right): f(x) \leq t\right\}
$$

is bounded since $X$ is a finite dimensional space and the function $f$ defined by (2.14) is l.s.c. on $X_{1}$ and, thus, $A$ is closed. Suppose to the contrary that there exist $t \in R^{1}$ and $\left\{x_{n}^{\prime}\right\} \subseteq X_{1}\left(\delta_{1}\right)$ such that $\left\|x_{n}^{\prime}\right\| \rightarrow+\infty$ and $f\left(x_{n}^{\prime}\right) \leq t$. From $\left\{x_{n}^{\prime}\right\} \subseteq X_{1}\left(\delta_{1}\right)$, we have

$$
d_{X_{0}}\left(x_{n}^{\prime}\right) \leq \delta_{1} .
$$

Thus,

$$
\max \left\{f\left(x_{n}^{\prime}\right), d_{X_{0}}\left(x_{n}^{\prime}\right)\right\} \leq \max \left\{t, \delta_{1}\right\},
$$

which contradicts condition (3.27).

Now, we show that if (iv) holds, then (VQVI) is type I LP well posed. Let $\left\{x_{n}\right\}$ be a type I LP approximating solution sequence of (VQVI). Then, there exist $\left\{\epsilon_{n}\right\} \subseteq R_{+}^{1}$ with $\epsilon_{n} \rightarrow 0$ and $z_{n} \in T\left(x_{n}\right)$ such that

$$
\begin{gathered}
\left\langle F\left(x_{n}\right), x^{\prime}-x_{n}\right\rangle+\epsilon_{n} \notin \operatorname{int} C, \quad \forall x^{\prime} \in S\left(x_{n}\right), \\
d_{X_{0}}\left(x_{n}\right) \leq \epsilon_{n}, \\
x_{n} \in S\left(x_{n}\right) .
\end{gathered}
$$


From (3.32) and (3.33), we can assume without loss of generality that $\left\{x_{n}\right\} \subseteq X_{1}\left(\delta_{1}\right)$. By Lemma 2.7, we can assume without loss of generality that

$$
\left\{x_{n}\right\} \subseteq\left\{x \in X_{1}\left(\delta_{1}\right): f(x) \leq 1\right\},
$$

where $f$ is defined by (2.14). By the level compactness of $f$ on $X_{1}\left(\delta_{1}\right)$, there exist a subsequence of $\left\{x_{n_{j}}\right\}$ of $\left\{x_{n}\right\}$ and $\bar{x} \in X_{1}\left(\delta_{1}\right)$ such that $x_{n_{j}} \rightarrow \bar{x}$. From this fact and (3.32), we have $\bar{x} \in X_{0}$. Since $S$ is closed and (3.33) holds, we also have $\bar{x} \in S(\bar{x})$. That is,

$$
\bar{x} \in X_{0} \cap X_{2}=X_{0}^{\prime}
$$

Furthermore, by Lemmas 2.7 and 3.6, we have

$$
f(\bar{x}) \leq \liminf _{n \rightarrow+\infty} f\left(x_{n_{j}}\right) \leq \limsup _{n \rightarrow+\infty} f\left(x_{n_{j}}\right) \leq 0 .
$$

We know that $f(\bar{x}) \geq 0$ by Lemma 2.6, so $f(\bar{x})=0$. This fact combined with (3.35) and Lemma 2.6 implies that $\bar{x} \in \bar{X}$.

Similarly, we can prove the next proposition.

Proposition 3.13. Let Assumption 1 hold. Further assume that one of the following conditions holds.

(i) There exists $0<\delta_{1}<\delta_{0}$ such that $X_{2}\left(\delta_{1}\right)$ is compact, where

$$
X_{2}\left(\delta_{1}\right)=\left\{x \in X_{1} \cap X_{2}: d_{K}(g(x)) \leq \delta_{1}\right\}
$$

(ii) the function $f$ defined by (2.14) is level compact on $X_{1} \cap X_{2}$,

(iii) $X$ is finite dimensional and

$$
\lim _{x \in X_{1} \cap X_{2},\|x\| \rightarrow+\infty} \max \left\{f(x), d_{K}(g(x))\right\}=+\infty
$$

(iv) There exists $0<\delta_{1}<\delta_{0}$ such that $f$ is level compact on $X_{2}\left(\delta_{1}\right)$ defined by (3.37). Then, (VQVI) is generalized type I LP well posed.

Remark 3.14. If $X$ is finite dimensional, then the "level-compactness" condition in Propositions 3.12 and 3.13 can be replaced by the "level-boundedness" condition.

Now, we consider the case when $Y$ is a normed space, $K$ is a closed and convex cone with nonempty interior int $K$ and let $e \in \operatorname{int} K$.

Let $t \geq 0$ and denote

$$
X_{3}(t)=\left\{x \in X_{1} \cap X_{2}: g(x) \in K-t e\right\}
$$

The next proposition follows immediately from Proposition 2.8(i), Lemma 3.6, and [12, Proposition 2.3(iv)]. 
Proposition 3.15. Let $Y$ be a normed space, let $K$ be a closed and convex cone with nonempty interior $\operatorname{int} K$ and $e \in \operatorname{int} K$. Let the set-valued map $S$ be closed and l.s.c on $X_{1}$. Assume that the solution set $\bar{X}$ of (VQVI) is nonempty. Further assume that there exists $t_{1}>0$ such that the function $f(x)$ defined by (2.14) is level compact on $\mathrm{X}_{3}\left(t_{1}\right)$, then (VQVI) is generalized type I LP well posed.

Remark 3.16. If $X$ is finite dimensional, then the level-compactness condition of $f$ can be replaced by the level boundedness of $f$.

\section{Acknowledgment}

This work is supported by the National Science Foundation of China.

\section{References}

[1] F. Facchinei and J.-S. Pang, Finite-Dimensional Variational Inequalities and Complementarity Problems, vol. 1-2 of Springer Series in Operations Research, Springer, New York, NY, USA, 2003.

[2] A. Bensoussan, "Points de nash dans le cas de fonctionnelles quadratiques et jeux différentiels linéaires à N personnes," SIAM Journal on Control and Optimization, vol. 12, pp. 460-499, 1974.

[3] J.-S. Pang and M. Fukushima, "Quasi-variational inequalities, generalized Nash equilibria, and multileader-follower games," Computational Management Science, vol. 2, no. 1, pp. 21-56, 2005.

[4] C. Baiocchi and A. Capelo, Variational and Quasivariational Inequalities, A Wiley-Interscience Publication, John Wiley \& Sons, New York, NY, USA, 1984.

[5] M. Fukushima, "A class of gap functions for quasi-variational inequality problems," Journal of Industrial and Management Optimization, vol. 3, no. 2, pp. 165-171, 2007.

[6] F. Giannessi, "Theorems of alternative, quadratic programs and complementarity problems," in Variational Inequalities and Complementarity Problems, R. W. Cottle, F. Giannessi, and J. L. Lions, Eds., pp. 151-186, John Wiley \& Sons, New York, NY, USA, 1980.

[7] G. Y. Chen and N. D. Yen, “On the variational inequality model for network equilibrium," Internal Report 3.196 (724), Department of Mathematics, University of Pisa, Pisa, Italy, 1993.

[8] L. C. Ceng, G. Y. Chen, X. X. Huang, and J.-C. Yao, "Existence theorems for generalized vector variational inequalities with pseudomonotonicity and their applications," Taiwanese Journal of Mathematics, vol. 12, no. 1, pp. 151-172, 2008.

[9] G. Y. Chen, X. X. Huang, and X. Q. Yang, Vector Optimization, Set-Valued and Variational Analysis, vol. 541 of Lecture Notes in Economics and Mathematical Systems, Springe, Berlin, Germany, 2005.

[10] A. N. Tikhonov, "On the stability of the functional optimization problem," USSR Computational Mathematics and Mathematical Physics, vol. 6, no. 4, pp. 28-33, 1966.

[11] E. S. Levitin and B. T. Polyak, "Convergence of minimizing sequences in conditional extremum problems," Soviet Mathematics Doklady, vol. 7, pp. 764-767, 1966.

[12] X. X. Huang and X. Q. Yang, "Generalized Levitin-Polyak well-posedness in constrained optimization," SIAM Journal on Optimization, vol. 17, no. 1, pp. 243-258, 2006.

[13] G. Beer and R. Lucchetti, “The epi-distance topology: continuity and stability results with applications to convex optimization problems," Mathematics of Operations Research, vol. 17, no. 3, pp. 715-726, 1992.

[14] A. L. Dontchev and T. Zolezzi, Well-Posed Optimization Problems, vol. 1543 of Lecture Notes in Mathematics, Springer, Berlin, Germany, 1993.

[15] J. P. Revalski, "Hadamard and strong well-posedness for convex programs," SIAM Journal on Optimization, vol. 7, no. 2, pp. 519-526, 1997.

[16] R. Lucchetti and J. Revalski, Eds., Recent Developments in Well-Posed Variational Problems, vol. 331 of Mathematics and its Applications, Kluwer Academic, Dodrecht, The Netherlands, 1995.

[17] T. Zolezzi, "Well-posedness criteria in optimization with application to the calculus of variations," Nonlinear Analysis: Theory, Methods E Applications, vol. 25, no. 5, pp. 437-453, 1995.

[18] T. Zolezzi, "Extended well-posedness of optimization problems," Journal of Optimization Theory and Applications, vol. 91, no. 1, pp. 257-266, 1996. 
[19] E. Bednarczuk, "Well-posedness of vector optimization problems," in Recent Advances and Historical Development of Vector Optimization Problems, J. Jahn and W. Krabs, Eds., vol. 294 of Lecture Notes in Economics and Mathematical Systems, pp. 51-61, Springer, Berlin, Germany, 1987.

[20] S. Deng, "Coercivity properties and well-posedness in vector optimization," RAIRO Operations Research, vol. 37, no. 3, pp. 195-208, 2003.

[21] X. X. Huang, "Extended well-posedness properties of vector optimization problems," Journal of Optimization Theory and Applications, vol. 106, no. 1, pp. 165-182, 2000.

[22] X.X. Huang, "Extended and strongly extended well-posedness of set-valued optimization problems," Mathematical Methods of Operations Research, vol. 53, no. 1, pp. 101-116, 2001.

[23] P. Loridan, "Well-posedness in vector optimization," in Recent Developments in Well-Posed Variational Problems, vol. 331 of Mathematics and Its Applications, pp. 171-192, Kluwer Academic, Dodrecht, The Netherlands, 1995.

[24] R. Lucchetti, Well-Posedness Towards Vector Optimization, vol. 294 of Lecture Notes in Economics and Mathematical System, Springer, New York, NY, USA, 1987.

[25] A. S. Konsulova and J. P. Revalski, "Constrained convex optimization problems-well-posedness and stability," Numerical Functional Analysis and Optimization, vol. 15, no. 7-8, pp. 889-907, 1994.

[26] X. X. Huang and X. Q. Yang, "Levitin-Polyak well-posedness of constrained vector optimization problems," Journal of Global Optimization, vol. 37, no. 2, pp. 287-304, 2007.

[27] Y.-P. Fang, N.-J. Huang, and J.-C. Yao, "Well-posedness of mixed variational inequalities, inclusion problems and fixed point problems," Journal of Global Optimization, vol. 41, no. 1, pp. 117-133, 2008.

[28] Y.-P. Fang, N.-J. Huang, and J.-C. Yao, "Well-posedness by perturbations of mixed variational inequalities in Banach spaces," European Journal of Operational Research, vol. 201, no. 3, pp. 682-692, 2010.

[29] M. B. Lignola and J. Morgan, " $\alpha$-well-posedness for Nash equilibria and for optimization problems with Nash equilibrium constraints," Journal of Global Optimization, vol. 36, no. 3, pp. 439-459, 2006.

[30] M. B. Lignola and J. Morgan, “Generalized variational inequalities with pseudomonotone operators under perturbations," Journal of Optimization Theory and Applications, vol. 101, no. 1, pp. 213-220, 1999.

[31] X. X. Huang, X. Q. Yang, and D. L. Zhu, "Levitin-Polyak well-posedness of variational inequality problems with functional constraints," Journal of Global Optimization, vol. 44, no. 2, pp. 159-174, 2009.

[32] L. C. Ceng, N. Hadjisavvas, S. Schaible, and J. C. Yao, "Well-posedness for mixed quasivariational-like inequalities," Journal of Optimization Theory and Applications, vol. 139, no. 1, pp. 109-125, 2008.

[33] M. B. Lignola, “Well-posedness and L-well-posedness for quasivariational inequalities," Journal of Optimization Theory and Applications, vol. 128, no. 1, pp. 119-138, 2006.

[34] X. X. Huang, B. Jiang, and J. Zhang, "Levitin-Polyak well-posedness of quasivariational inequality problems with functional constraints," Natural Science Jourmal of Xiangtan University, vol. 30, pp. 1-11, 2008.

[35] B. Jiang, J. Zhang, and X. X. Huang, "Levitin-Polyak well-posedness of generalized quasivariational inequalities with functional constraints," Nonlinear Analysis: Theory, Methods \& Applications, vol. 70, no. 4, pp. 1492-1503, 2009.

[36] L. Q. Anh, P. Q. Khanh, D. T. M. Van, and J.-C. Yao, "Well-posedness for vector quasiequilibria," Taiwanese Journal of Mathematics, vol. 13, no. 2, pp. 713-737, 2009.

[37] X.X. Huang and X. Q. Yang, "Levitin-Polyak well-posedness of vector variational inequality problems with functional constraints," Numerical Functional Analysis and Optimization, vol. 31, no. 4, pp. 440-459, 2010.

[38] S. J. Li and M. H. Li, "Levitin-Polyak well-posedness of vector equilibrium problems," Mathematical Methods of Operations Research, vol. 69, no. 1, pp. 125-140, 2009.

[39] G. Wang, X. X. Huang, J. Zhang, and G. Y. Chen, "Levitin-Polyak well-posedness for vector equilibrium problems with functional constraints," Acta Mathematica Scientia, vol. 30, no. 5, pp. 14001412, 2010.

[40] X. X. Huang, K. L. Teo, and X. Q. Yang, "Calmness and exact penalization in vector optimization with cone constraints," Computational Optimization and Applications, vol. 35, no. 1, pp. 47-67, 2006.

[41] M. Furi and A. Vignoli, "About well-posed minimization problems for functionals in metric spaces," Journal of Optimization Theory and Applications, vol. 5, pp. 225-229, 1970. 